Itinéraires Itinéraires

Littérature, textes, cultures

2011-1| 2011

Les Mémoires, une question de genre?

\title{
« Je suis femme, il est vrai »: Mémoires et code féminin chez la duchesse d'Abrantès
}

\section{Damien Zanone}

\section{OpenEdition}

1 Journals

\section{Édition électronique}

URL : http://journals.openedition.org/itineraires/1621

DOI : 10.4000/itineraires. 1621

ISSN : 2427-920X

Éditeur

Pléiade

\section{Édition imprimée}

Date de publication : 1 avril 2011

Pagination : 73-84

ISBN : 978-2-296-13692-2

ISSN : 2100-1340

Référence électronique

Damien Zanone, « « Je suis femme, il est vrai » : Mémoires et code féminin chez la duchesse

d'Abrantès », Itinéraires [En ligne], 2011-1 | 2011, mis en ligne le 01 avril 2011, consulté le 25 juin 2020

URL : http://journals.openedition.org/itineraires/1621; DOI : https://doi.org/10.4000/itineraires.1621

\section{(c) $(1) \odot$}

Itinéraires est mis à disposition selon les termes de la licence Creative Commons Attribution - Pas d'Utilisation Commerciale - Pas de Modification 4.0 International. 


\title{
« Je suis femme, il est vrai » : Mémoires et code féminin chez la duchesse d'Abrantès
}

\begin{abstract}
Approximately one quarter of the large number of memoirs published during the first half of the nineteenth century were produced by, or are attributable to, women. How, in an era so reluctant to let the word of women into the public arena, did works penned by women become recognized as "Historical memoirs"? The study of the memoirs of the Duchess of Abrantès provides an answer: by implementing rules for expression (e.g. the use of conversational and of emotional words) that seem to be generally absent from the writings of male memoirists.
\end{abstract}

Keywords : woman, memoirs, testimony, Napoleon, conversation

Mots clés : femme, Mémoires, témoignage, Napoléon, conversation

Un quart environ du grand nombre de Mémoires publiés durant la première moitié du XIX ${ }^{\mathrm{e}}$ siècle est dû ou attribué à des femmes. La proportion est remarquable pour des écrits qui se font valoir comme historiques, en un temps où la possibilité d'intervention publique des femmes est strictement contrôlée. L'étude que j'ai consacrée à ce moment saillant de l'histoire de cette forme d'écrit (Écrire son temps. Les Mémoires en France de 1815 à $1848^{1}$ ) s'est employée à décrire un phénomène éditorial et à en comprendre la signification sous l'aspect d'une fable exemplaire faisant se succéder « grandeur » et « décadence ». Au début des années 1820, la publication de Mémoires enthousiasmait le public lettré et ravissait les esprits sérieux (les historiens Thierry et Guizot ou encore Chateaubriand qui, dès le Génie du christianisme, s'était posé en esthète des Mémoires) : c'est le temps des grandes collections savantes par lesquelles on remet en circulation les Mémoires des siècles passés et où l'on adoube, grâce à eux, ceux qui viennent pour le temps présent. À partir du milieu des années

1. Damien Zanone, Écrire son temps. Les Mémoires en France de 1815 à 1848, Lyon, Presses universitaires de Lyon, 2006. 
1820, les auteurs sont de plus en plus nombreux à publier leurs Mémoires de leur vivant, sans les mettre à l'abri de collections savantes. Enfin, dès la fin de cette décennie, on constate la prolifération de faux qui fait la fortune des uns et le scandale des autres, qui destitue ces écrits du point de vue sérieux (historien) et les déporte dans les parages du roman : ceux-ci se révèlent très proches.

Or, force est de constater que la publication d'un certain nombre de Mémoires dus ou attribués à des femmes n'a pas peu contribué à cette évolution que les esprits graves et masculins considéraient comme une dégradation au moment même où ils y assistaient, mais que l'on peut tout aussi bien envisager, hors axiologie, d'un point de vue analytique et descriptif. Dans quelle mesure la reconnaissance d'écrits de femmes en tant que « Mémoires historiques » était-elle possible? On cherchera à répondre à cette question par l'étude privilégiée de trois de ces textes qui, à leur publication, ont eu un écho important par leur manière de s'inscrire dans le genre en fixant pour un moment, dans le discours commun, l'idée de ce que des Mémoires doivent être ou ne doivent pas être. Il s'agit des Mémoires de Madame de Genlis (1825), des Mémoires d'une Contemporaine (1827-1828) et des Mémoires de la duchesse d'Abrantès (1831-1835); ce dernier ouvrage nous retiendra particulièrement. À leur occasion, nous mettrons en valeur trois aspects qui ne se rencontrent pas de la même façon chez les mémorialistes hommes : la conquête du témoignage, la maîtrise de la conversation, la modulation d'une parole affective.

\section{Trois Mémoires de femmes}

Commençons par une courte présentation monographique de chacun de ces ouvrages.

Mme de Genlis est un personnage omniprésent sur la scène mondaine, littéraire et même politique depuis le milieu des années 1760, quand en 1825, à soixante-dix-neuf ans, elle publie ses Mémoires². Avec ceux-ci, elle prétend justifier une carrière à la trajectoire très contestée et apporter un témoignage de première main sur les milieux qu'elle a fréquentés : la « bonne compagnie » des vingt années qui précèdent la Révolution ainsi que les cercles littéraires et artistiques sous l'Empire. Cette publication est une date marquante pour l'histoire du genre : l'auteur se flatte d'être le premier auteur à publier ses Mémoires de son vivant et, pour l'occasion, le libraireéditeur Ladvocat décide d'inscrire les Mémoires parmi les spécialités de son catalogue; il lance l'ouvrage avec un grand fracas publicitaire, démarche nouvelle pour l'époque et donc encore susceptible d'étonner. Aussi bien pour leur contenu que pour ce mode de publication, ces Mémoires sont

2. Mme de Genlis, Mémoires de Madame de Genlis sur le dix-huitième siècle et la Révolution française, Paris, Ladvocat, 1825,8 vol. in- $8^{\circ}$. 
le symptôme de nouvelles mœurs éditoriales : on les publie parce qu'on attend d'eux du scandale (l'ouvrage est riche en anecdotes et fait le portrait moqueur d'un grand nombre de contemporains).

Publiés sans nom d'auteur en 1827, les Mémoires d'une Contemporaine ${ }^{3}$ réalisent a priori la prouesse d'être des Mémoires anonymes - mais l'on sait bien que cette forme avait été employée sans relâche par le roman au $\mathrm{XVIII}^{\mathrm{e}}$ siècle. La confection d'un tel ouvrage - une pure fabrication commerciale à laquelle se prêtent un ensemble de faiseurs («teinturiers ») réunis par Ladvocat - et plus encore son succès en disent long sur l'engouement de l'époque en faveur des Mémoires. Bourrés d'anecdotes qui prétendent dévoiler, sur un ton léger et plaisant, les coulisses de l'histoire récente, ils sont supposés l'œuvre d'une aventurière plus ou moins courtisane, Ida Saint-Elme, qui se prête au jeu en acceptant de passer pour le référent de la « Contemporaine ». Il est significatif que cette entreprise réussisse sur la seule foi d'un titre dont le mérite essentiel et presque exclusif est d'afficher et d'annoncer une parole féminine. Celle-ci est prise en charge dans le texte avec l'efficacité d'un rôle. Ainsi témoigne un commentateur de l'époque :

\footnotetext{
Beaucoup plus de lecteurs qu'on ne pense se sont trouvés arrêtés dès le titre de ce volumineux ouvrage. "Qu'est-ce qu'une contemporaine? se demandaient-ils. Est-ce une dignité, une profession? Est-on contemporaine comme on est ambassadrice, cantatrice ou femme de lettres? » Il ne faut pas se moquer de la niaiserie de ces lecteurs; ils s'en sont tenus au dictionnaire, et, d'après l'unique acception du mot, ils ont trouvé que, sans sortir de France seulement, le nombre de leurs contemporaines, filles, femmes ou veuves, s'élevait à seize millions! Qu'a donc celle-ci qui la distingue des autres? C'est ce que l'on ne tarde pas à apprendre en tournant la page ${ }^{4}$.
}

En effet, il faut tourner la page : la «Contemporaine» est un être de papier. L'idée n'est valable qu'une seule fois mais elle marche. C'est sans doute la raison pour laquelle d'autres Mémoires conçus sur ce modèle (par exemple, en 1829-1830, Mémoires d'une femme de qualité) ont eu moins de succès.

Les Mémoires de la duchesse d'Abrantès ${ }^{5}$ ont conservé une petite notoriété dans l'histoire littéraire, parce que leur auteur fut une grande amie de Balzac et qu'on a longtemps dit que celui-ci avait dû contribuer à leur rédaction, ce qui n'est généralement plus l'avis dominant aujourd'hui (même si le débat existe toujours).

3. Mémoires d'une Contemporaine ou Souvenirs d'une femme sur les principaux personnages de la République, du Consulat, de l'Empire, Paris, Ladvocat, 1827-1828, 8 vol. in- $8^{\circ}$. 4. Charles-Louis de Sévelinges, La Contemporaine en miniature, ou Abrégé critique de ses Mémoires, Paris, Dentu, 1828, p. 1.

5. Laure d'Abrantès, Mémoires de Madame la Duchesse d'Abrantès, Souvenirs historiques sur Napoléon, la Révolution, le Directoire, le Consulat, l'Empire et la Restauration, Paris, Ladvocat, 1831-1835, 18 vol. in-16. 
Ce qui autorise la duchesse d'Abrantès à publier des Mémoires en dix-huit volumes, c'est d'avoir été une personne proche de Napoléon, une figure notable de son entourage familier (sa famille est liée à celle des Bonaparte, Napoléon protège la jeune fille et lui fait épouser le général Junot, duc d'Abrantès). À en croire la mémorialiste, elle aurait été à toutes les époques l'amie la plus proche et la confidente inlassable de celui qui s'est fait empereur. Elle mène sous l'Empire une vie sociale très brillante; retirée et isolée ensuite, elle échappe à la misère grâce à divers travaux d'écriture dont ses Mémoires sont le plus notable.

Le prétexte à leur publication est, dit-elle, le souci de laver la mémoire de son époux et de réhabiliter la figure de Napoléon : toutes deux se trouveraient salies par d'autres Mémoires et demanderaient à être défendues. La mémorialiste se présente donc comme une femme qui intervient pour sauver l'honneur menacé des deux hommes qui lui furent les plus chers.

\section{Être femme et témoigner}

La mémoire historique qui s'énonce au féminin ne peut pas être identique à celle qui s'énonce au masculin : elle ne le peut pas parce qu'elle ne le doit pas. Cette mémoire au féminin est amenée à mettre en œuvre des codes d'expression qui lui sont propres et qui déterminent certains effets.

\footnotetext{
Je suis femme, il est vrai, mais il est absurde aujourd'hui de récuser le témoignage des femmes et leur opinion. Nous sommes maintenant ce que nous fûmes toujours, mais nous avons, de plus, un développement de facultés qui existaient bien autrefois dans nous, et que nos habitudes, notre éducation, et surtout le préjugé paralysaient et repoussaient même. Notre sort est tout autre. Je ne parle pas ici des absurdités plus qu'absurdes que disent les saint-simoniens. Je prétends seulement que la femme possède une âme forte, un cœur courageux, des vertus patriotiques et civiques, je prétends qu'elle peut tout ce que peut un homme par la force et la puissance de la volonté. Quant à moi, la force physique est la seule que je reconnaisse, comme obstacle à ma détermination de faire. [...] Eh quoi! serait-ce donc parce qu'un homme lancera une barre de fer vingt pieds plus loin que moi, que je serai jugée indigne de comprendre et d'agir? Mais ce serait réduire l'homme en général à l'état de brute 6 .
}

Ainsi donc une bonne fois, et alors que ses Mémoires sont déjà très avancés, la duchesse d'Abrantès éclate et dit sa lassitude de devoir constamment, tout au long de sa narration, trouver des arguments et autres tactiques de discours pour faire valoir la validité de son témoignage. La légitimité de Mémoires à venir au public tient dans le fait d'apporter des révélations sur les événements dont on a été témoin. Avoir « tout vu», « tout entendu» : tel est le mérite, qu'idéalement, les mémorialistes veulent se faire reconnaître. C'est à ce titre qu'ils peuvent espérer retenir l'attention des lecteurs.

6. Laure d'Abrantès, op. cit., vol. VI, p. 395. 
D'où l'usage qui consiste à mettre en avant ses fonctions comme propices à la meilleure observation possible : ministre, ambassadeur, directeur de la police... Ces fonctions échappent, au XIX ${ }^{\mathrm{e}}$ siècle, à la carrière féminine. Les seuls postes accessibles aux femmes, et qui donneraient une sorte de patente officielle à la mémoire historique, sont très subalternes : « femme de chambre » par exemple, quand on l'a été auprès de Marie-Antoinette ou de Joséphine. Les femmes mémorialistes doivent donc convaincre les lecteurs de la valeur de la position d'observation qu'elles ont eue mais qu'aucun titre officiel ne sanctionne. Une « femme de papier» comme la Contemporaine peut faire valoir qu'elle a été la maîtresse de tel ou tel grand homme : mais seule la fiction peut faire assumer sans déshonneur le statut de courtisane. La duchesse d'Abrantès, qui n'a pas cette ressource, se fait valoir comme meilleure amie de Napoléon, et cela depuis l'enfance. Mais qu'est-ce à dire? Napoléon Bonaparte a-t-il cultivé des amitiés féminines? Etait-il enclin à causer haute politique et stratégie militaire avec une femme? Si le fait est, le secret en a été bien gardé! La duchesse d'Abrantès est obligée, pour rendre crédible son discours, de revendiquer pour ellemême un statut d'exception qu'elle construit patiemment, dès que son récit y invite. Ainsi, quand elle évoque le souvenir d'une conversation où l'empereur se serait exprimé devant un groupe de femmes dont elle était :

En relisant ces mots : le premier consul nous dit, j'espère que l'on ne me croira pas assez sotte - je me sers de ce mot - pour raconter que le premier consul nous faisait l'honneur de nous admettre à ses hautes conversations [...]. Si je le faisais, d'abord mes contemporains sauraient très bien que cela ne fut jamais; et nos petits-neveux, d'après ce qu'ils pourront recueillir de Napoléon, sauront très bien aussi qu'il n'était pas très causeur avec les femmes. Mais je dois employer cette formule, il nous dit, parce que, en effet, il parlait dans le salon de Mme Bonaparte où nous étions toutes, et si quelques-unes de ces dames ne l'écoutaient pas, quant à moi, je réponds qu'il me parlait, car je suivais mot à mot tous ses discours et il m'est quelquefois arrivé de faire une telle attention à ce qu'il avait dit, qu'en rentrant chez moi j'écrivais la conversation que j'avais entendue sans qu'un seul mot y manquât ${ }^{7}$.

Non seulement la justification est apportée, le témoignage maintenu, mais celui-ci est encore renforcé par la revendication d'une précision exhaustive : des notes ont été prises. La mémorialiste revient à plusieurs reprises sur ce sujet pour désarmer les possibles moqueries anti-féminines de ses lecteurs. Sans doute le grand homme «n'était pas autrement gracieux avec les femmes et ce n'eût certainement pas été pour cause de choses de la nature de celles que je viens de rapporter qu'il se serait assis près de l'une de nous ${ }^{8} »$. Mais tantôt il distingue du lot son amie Laure (« il me savait

7. Ibid., vol. III, p. 263.

8. Ibid., vol. IV, p. 538. 
quelque gré d'écouter avec cette attention; il me le disait $\left.{ }^{9} \gg\right)$; tantôt son mépris intellectuel pour un sexe aussi insignifiant l'empêche même de se soucier de ce qu'il dit devant lui :

Ce n'est même qu'à l'aide de ma nullité de femme que je recueillais des paroles dites par lui, soit lorsqu'il se promenait dans l'allée qui longe le château de la Malmaison, [...] soit pendant qu'il parcourait la petite galerie à côté du salon ${ }^{10}$.

Ainsi, à force de répondre à des objections qui veulent invalider son titre au témoignage historique sous le prétexte de sa condition féminine, Laure d'Abrantès finit par transformer l'obstacle en atout. Elle prétend à un privilège, par exemple, pour ce qui concerne «l'intime intérieur de Joséphine » : il semble qu'elle seule, hormis Napoléon - et les femmes de chambre - ait pénétré ce sanctuaire. C'est aussi comme femme qu'elle aurait entretenu de bonnes relations d'amitié avec les sœurs Bonaparte. Elle se dit «l'esprit observateur » et en tout cas doué d'une «vive curiosité » :

Cette dénomination conviendrait peut-être mieux à l'esprit d'une femme, m'observera-t-on? Soit; ce sera comme l'on voudra. [...] Mon esprit sera curieux et point observateur, si l'on trouve qu'une femme ne doive pas s'aviser d'usurper, pour ce qui la regarde, des noms aussi respectables que celui d'observateur donné à son esprit ${ }^{11}$.

Ainsi, et volontiers avec humour, le rôle féminin, obstacle supposé du témoignage, en devient une garantie. La narratrice semble s'adresser à la doxa masculine en lui disant à peu près : « Vous ne voulez pas de nous comme observatrices? Soit, nous serons curieuses!» Dont acte, quand il s'agit de rapporter à une autre occasion une audience qu'elle obtint auprès de Louis XVIII et une question qu'elle se permît de lui poser sur un point bien accessoire : «Si je n'avais pas fait cette question, je n'aurais pas été femme $e^{12}$.» Cumulant les avantages des deux sexes (attentive aux enjeux politiques; autorisée à fréquenter la chambre de Joséphine), la mémorialiste peut finalement revendiquer : «Je ne crains donc pas d'affirmer que, de toutes les personnes qui ont parlé de l'empereur, je suis la seule qui puisse donner des détails aussi complets ${ }^{13}$. »

Mais cette réussite n'est acquise qu'au titre d'exception : une femme peut la revendiquer, mais une seule. Pour les autres, la position d'énonciation est à travailler dans une autre voie : le ton de la conversation en est une, celui de la plainte sensible en est une autre.

9. Laure d'Abrantès, op. cit., vol. III, p. 263.

10. Ibid., vol. IV, p. 538.

11. Ibid., vol. III, p. 146.

12. Ibid., vol. II, p. 443.

13. Ibid., vol. I, p. 9. 


\section{La parole des salons, la conversation}

Dans le XVIII siècle où était née et où avait grandi Madame de Genlis, la conversation était une pratique éminente, elle s'y était affinée comme un art dans les salons, lieux d'un règne supposé du féminin. Cet « art », parfois mythifié au point d'être tenu pour un genre littéraire ${ }^{14}$, aurait sa poétique au caractère composite : s'y mêlent portraits, pointes morales, digressions, improvisations, mélange des tons...

Ces caractères sont ceux que présentent les Mémoires de la duchesse d'Abrantès. Les lire peut parfois donner l'impression de se mettre à l'écoute de ce qui se disait dans un salon bonapartiste sous la Restauration : sous cet aspect, on peut les apprécier comme une émanation collective (le compte rendu des conversations de certains jours). À telle page, il semble que la mémorialiste parle de Napoléon à son lecteur comme elle le ferait, chez elle, à l'un de ses hôtes :

Une particularité qui ne m'étonne pas, mais que je prie de remarquer, c'est que tous les beaux talents de l'époque sont éloignés non seulement de mal penser, mais de mal parler de Napoléon. Ils ont senti le ridicule de la position de l'homme qui ne le comprendrait pas. Voyez Chateaubriand, Lamartine, Victor Hugo, Casimir Delavigne, l'abbé de Lamennais, ces hommes-là ont jugé le colosse. Ils ont vu et blâmé ses fautes, mais reconnu ses qualités. [...] La médiocrité seule l'a méconnu ${ }^{15}$.

La crainte du « ridicule » mondain semble devenue l'argument ultime qui commande la manière de juger le grand homme. Les noms des sommités littéraires du moment sont convoqués comme ceux d'arbitres du bon goût en la matière; ils sont là pour réclamer l'approbation qui doit venir de l'interlocuteur.

Entre ces Mémoires, les salons et les journaux, un espace de délibération unique est ouvert, sans frontières, où circulent les discours. La publication de Mémoires prend ainsi valeur d'un geste mondain où l'écriture est socialisée comme une parole échangée. Il est significatif que l'auteur, après la publication de ses Mémoires, ait prolongé son aventure d'écriture par une Histoire des salons de Paris. Tableaux et portraits du grand monde, sous Louis XVI, le Directoire, le Consulat et l'Empire, la Restauration et le règne de LouisPhilippe I ${ }^{e r}$, publiée en six volumes chez Ladvocat en 1837-1838. La complémentarité de ces deux rôles a bien été mise en valeur par Loïc Chotard dans l'anthologie où il fait se croiser des extraits des deux ouvrages ${ }^{16}$.

14. Ainsi le fait Marc Fumaroli, qui reprend la leçon de Sainte-Beuve : Marc Fumaroli, « La conversation », dans Pierre Nora (dir.), Lieux de mémoire. La France, Paris, Gallimard, coll. «Quarto », 1997, p. 3617-3675.

15. Laure d'Abrantès, Mémoires, op. cit., vol. III, p. 216.

16. Laure d'Abrantès, Salons révolutionnaires, éd. Loïc Chotard, Paris, Éditions FranceEmpire, 1989. 
La dimension conversée et « salonnière » propre à certains Mémoires de femmes est également sensible dans ceux de Madame de Genlis. Quand, dans leur dernier tiers (à partir du sixième volume), l'auteur a achevé le récit de son existence depuis sa naissance jusqu'au moment présent, elle décide... de ne pas achever ses Mémoires, mais de continuer avec la rédaction d'un ouvrage qu'il lui plaît d'écrire. S'ensuivent alors encore deux volumes qui ne sont plus que de causerie. Le récit des Mémoires se transforme en une sorte de chronique du moment présent : on y donne son avis sur le livre du moment (par exemple Ourika de Mme de Duras), on y tient le carnet mondain des naissances, des mariages et des décès. Les Mémoires procurent alors, semble-t-il, l'écho sonore d'un microcosme social.

Enfin, il faut sans doute expliquer par le modèle féminin de la conversation l'énonciation légère, badine même, que peuvent s'autoriser les Mémoires de femmes : c'est une façon de se mettre en valeur vis-à-vis du ton sérieux, à l'autorité faite de gravité, dont se prévalent les Mémoires d'hommes. Ce phénomène concerne tout particulièrement les Mémoires d'une Contemporaine et les Mémoires d'une femme de qualité, c'est-àdire des Mémoires attribués à des femmes mais rédigés par des hommes. Les "mercenaires de la plume» payés pour écrire ces textes font des "Mémoires de femmes », anticipent sur l'attente que l'on doit avoir, selon eux, vis-à-vis de tels textes : chargés de créer de toutes pièces une énonciation féminine, ils s'en acquittent par la caricature. Que font-ils? Ils imposent le ton du bavardage écervelé, badin, rapide, superficiel. Le suprême de cet art consiste à dire l'impossibilité où l'on est d'ordonner son propos : un charmant désordre doit prévaloir, qu'il serait bien dommage de ne pas goûter. Voici comment la « Contemporaine » justifie la composition erratique de ce qu'elle donne à lire :

\footnotetext{
Mon Dieu! je suis écrivain aussi désordonné que femme étourdie. Mes Mémoires ressemblent involontairement à mon existence et à mon caractère. Je suis au milieu des événements, et je les retrace bien moins suivant leur importance réelle que d'après l'impression individuelle que j'en ai ressentie ${ }^{17}$.
}

Être femme, sous la plume des scripteurs masculins qui la font s'exprimer, c'est être « femme étourdie » : elle peut dont être une narratrice fantasque. Le modèle une fois posé, il est rendu disponible pour une mémorialiste de chair et d'os : ainsi la duchesse d'Abrantès, qui publie ses Mémoires quatre ans après que la «Contemporaine » a donné les siens. Lorsqu'elle évoque les partisans du duc d'Orléans qui, au début de la Révolution, souhaitaient faire de celui-ci un monarque à l'anglaise et disaient « qu'il fallait habiller la France à l'anglaise » : 
Cela pouvait être bon jusqu'à un certain point, mais un calque pris sur la Constitution anglaise n'est pas ce qu'il nous faut. De même qu'une femme française ne sera pas bien mise avec des modes purement britanniques que n'auront pas rectifiées sa grâce et son bon goût, parce qu'elle n'aura pas ce teint de crème, ces yeux de lapis, ces cheveux de soie de Piémont qui sont généralement le partage d'une femme anglaise; de même aussi notre nation ne doit pas être gouvernée d'après les mêmes errements et recevoir les mêmes lois politiques que l'Angleterre ${ }^{18}$.

Le détour par les chiffons est allègrement avoué parce qu'il est donné pour garantir l'intelligence féminine en politique : c'est une compétence à parler du monde en se fondant sur un rapport concret à la matière même des choses. La valeur de Mémoires comme ceux de Mme de Motteville, dit la duchesse d'Abrantès à une autre occasion, est de prendre grand soin de donner des détails vestimentaires sur les personnages qu'elle décrit : « dans un ouvrage du genre des Mémoires, l'excès des détails qui donnerait la mort à un autre sujet, peut seul produire cet effet » de rendre sensible l'Histoire comme une réalité à laquelle on donne vie ${ }^{19}$. George Sand ne dira pas autrement dans Histoire de ma vie quand elle invite à considérer que l'histoire entière d'une époque peut être reconstituée à partir du moindre bout de tissu.

Ces exemples font cependant entendre que le modèle de la conversation ne prétend plus, au XIX ${ }^{\mathrm{e}}$ siècle, à la dignité d'art supérieur mais, dévalué comme bavardage, autorise toutes les désinvoltures.

Une autre modalité d'énonciation est autorisée aux femmes qui s'emploient à exprimer une mémoire historique : c'est celle des affects, du tremblement sensible.

\section{La parole des affects}

Si ce type de parole est autorisé, c'est qu'il est codifié : la parole féminine y est cadrée par des normes mises au point dans des modèles littéraires, en particulier le roman sentimental et le mélodrame théâtral. Le ton approprié est celui d'une emphase des sentiments qui vise au pathos: il s'agit d'émouvoir par des larmes contagieuses qui atteindront le lecteur. La parole est tout entière chargée d'affectivité : cette parole sensible, parole des affects, est celle qui, avec la conversation, est censée être l'apanage des femmes. Pour autant, elle n'est pas interdite aux hommes, comme Rousseau, Musset ou Lamartine.

La duchesse d'Abrantès est la mémorialiste qui recourt le plus volontiers à cette parole des affects : son écriture se fait volontiers vibrante, pour exprimer les événements qui l'ont touchée, et veut restituer sur un

18. Laure d'Abrantès, Mémoires, dans Salons révolutionnaires, éd. L. Chotard, op. cit., p. 190.

19. Laure d'Abrantès, Mémoires, op. cit., vol. II, p. 360. 
mode affectif sa participation à l'histoire nationale. Le pathos fonde une rhétorique : ressentant intensément les malheurs publics, la mémorialiste a beaucoup pleuré; ses larmes sont données pour preuve de l'authenticité de son caractère et de son récit. Dans son ouvrage qui est tout entier attaché à exalter la figure de Napoléon, la narration du déclin de l'Empire, dans les derniers volumes, prend l'aspect d'un long lamento. L'annonce de la mort du héros en est évidemment le point culminant :

Oh! que j'ai longtemps souffert, bien souffert, mon Dieu! Je ne portais pas le deuil ostensiblement en ayant une élégante robe noire un jour de première représentation à l'Opéra, et disant: " C'est pour l'anniversaire du 5 mai 1821! » mais lorsque me promenant dans les bois solitaires qui entouraient la demeure que j'avais choisie en quittant un monde qui avait l'impudeur de ne plus entourer de flatteries celle qui ne pouvait plus lui offrir les mêmes joies dans sa maison, lorsque je me demandais à moimême ce qu'étaient devenus tant d'amis véritables et que la réponse me venait du tombeau, oh! c'est alors que mon âme se brisait, et que je pleurais avec un déchirement de cœur qui m'a fait concevoir qu'on pouvait mourir d'une peine profonde ${ }^{20}$.

Dans cette longue phrase, la représentation de la réaction à l'événement est particulièrement soignée. C'est par les affects, est-il sous-entendu, que se manifeste le mieux la nature de la présence de chacun à l'histoire : c'est par eux que cette dernière se trouve personnalisée. La duchesse d'Abrantès accorde une mise en scène radicale à la singularité de la peine dont l'accable la mort de Napoléon : les «bois solitaires » ne sont pas assez grands pour lui faire mesurer l'étendue de cette perte et en aucun cas ils ne sauraient égarer le sens de cette douleur. L' « héroïne » s'est peut-être abstenue d'arborer un grand deuil à l'Opéra, mais quelle compensation ne se donne-t-elle pas dans ses Mémoires!

Comme femme, la mémorialiste a le droit aux larmes et aux cris. Ces pleurs ne viennent pas, précise-t-elle, de raisons superficielles, comme la perte de sa fortune (« jamais les pertes de ce genre n'ont eu le pouvoir de me faire jeter une larme $\left.{ }^{21} \gg\right)$. Les seuls malheurs qui peuvent l'atteindre et l'émouvoir vraiment sont ceux de la nation et aussi, puisqu'elle est femme, ceux qui la touchent en tant qu'épouse, fille et mère. Prenons la manière dont elle construit la représentation de la mort de son père : ces pages enfiévrées sont un sommet de mélodrame. La scène est à Paris pendant la Révolution, les rues grondent de l'émeute du 13 vendémiaire qu'on voudrait cacher au mourant :

Mon père était à l'agonie. Il criait, il pleurait. Jamais, non, jamais je ne souffrirai ce que j'ai souffert durant cette affreuse nuit. [...] Nous avions songé, d'abord, à lui parler d'une fête, de salves de réjouissance. Comme il

20. Laure d'Abrantès, Mémoires, op. cit., vol. IX, p. 102.

21. Ibid., vol. IX, p. 103. 
était extrêmement affaibli par une maladie longue et douloureuse, peut-être serait-on parvenu à le lui faire croire, sans l'indiscrétion d'une garde. Enfin, il savait tout. [...] J'aimais mon père avec une extrême tendresse; j'adorais ma mère! Je voyais l'un mourant des coups du canon qui retentissait, tandis que l'autre, étendue sur le pied du lit mortuaire, semblait prête à le suivre. Il y a des souvenirs éternels. Ceux de cette horrible nuit et de ces deux journées resteront incisés dans mon cœur avec un fer brûlant ${ }^{22}$.

Le registre pathétique se donne ici plein droit, il porte témoignage d'une expérience dont, par ailleurs, l'auteur fait un bilan qui concerne son sexe tout entier : " la gravité des événements » traversés dans le jeune âge amène cette mémorialiste née en 1784 à risquer, dès le début de ses Mémoires, le constat collectif : «je pense qu'à cet égard il en est de même de toutes les femmes de mon âge : nous n'avons eu ni enfance ni adolescence ${ }^{23} \gg$.

\section{Conclusion}

En élisant comme objet de réflexion la manière propre dont les femmes s'emparent de l'écriture de la mémoire historique, on est certes amené à constater que cette manière a des caractères spécifiques, mais surtout à se dire qu'il n'y a rien là d'étonnant : dans les Mémoires, les femmes qui écrivent ne font que reprendre les modes d'expression qui leur sont autorisés par ailleurs, parce que la tradition morale et littéraire les a codés comme féminins. Les registres auxquels elles ont droit sont donc, sans surprise, ceux de la conversation (qui peut confiner au bavardage) et de la parole affective (qui peut aller jusqu'à la sensiblerie).

Ce qui est remarquable, en revanche, c'est que le traitement auquel ces auteurs soumettent la mémoire historique va finalement l'emporter en contaminant progressivement, et très vite à vrai dire, le mode d'expression général des Mémoires. La conversation alerte, enjouée et parfois moqueuse; l'engagement affectif de la narration qui ne recule pas devant les effets pathétiques : finalement, c'est cela à quoi les lecteurs de Mémoires de l'époque ont très vite pris goût, dans les années 1820 et 1830 , c'est cela qu'ils ont aimé lire. Cela, plutôt que les considérations savantes ou méditations sur l'organisation des États, la stratégie militaire ou la philosophie de l'histoire, pour lesquelles les hommes se voulaient seuls compétents. Redisons combien il est notable que, dans la courte histoire qu'on peut faire des Mémoires de cette période, ceux que publient des femmes (réelles ou supposées) marquent les dates charnières : les Mémoires de Madame de Genlis qui commencent l'usage de publier de son vivant des ragots mondains. Les Mémoires d'une Contemporaine qui font dériver ces écrits du témoignage historique vers l'invention fictionnelle; les Mémoires

22. Laure d'Abrantès, Mémoires, op. cit., vol. I, p. 311.

23. Ibid., vol. I, p. 4. 
de la duchesse d'Abrantès viennent avec un regard déjà rétrospectif sur l'ensemble d'une production, pour y trier le bon grain de l'ivraie au nom de la vérité. Les esprits «sérieux »- masculins - ont évidemment déploré le phénomène, condamné la tendance de plus en plus marquée des Mémoires à aller vers le bavardage anecdotique et l'énonciation émotive. Mais le phénomène avait son dynamisme propre : et ce sont des mémorialistes femmes qui ont le mieux compris que l'attrait des Mémoires était finalement moins dans la construction d'un savoir historique que dans la satisfaction d'un goût de fabulation.

Damien Zanone

Université catholique de Louvain 